\title{
ON THE CHARACTERISTIC ROOTS OF TOURNAMENT MATRICES
}

\author{
BY ALFRED BRAUER ${ }^{1}$ AND IVEY C. GENTRY ${ }^{1}$
}

Communicated by Gian-Carlo Rota, July 15, 1968

A tournament matrix $A=\left(a_{i j}\right)$ of order $n$ is a matrix of zeros and ones whose main diagonal elements are zeros and all other elements satisfy $a_{i j}+a_{j i}=1$ for $i \neq j$. See, for instance [5].

Such matrices have recently been studied in a large number of papers. But not much seems to be known about their characteristic roots. Since they are nonnegative matrices whose two greatest rowsums are less than or equal to $n-1$ and $n-2$, respectively, it follows from [2] that they lie in the interior or on the boundary of the circle

$$
|z| \leqq((n-1)(n-2))^{1 / 2} \text {. }
$$

In this paper, this result will be improved.

THEOREM. Let $A$ be a tournament matrix of order $n$ with characteristic roots $\omega_{1}, \omega_{2}, \cdots, \omega_{n}$, and $R\left(\omega_{v}\right)$ the real part of $\omega_{p}$. Assume that

$$
\left|\omega_{1}\right| \geqq\left|\omega_{2}\right| \geqq \cdots \geqq\left|\omega_{n}\right| \text {. }
$$

Then

$$
-\frac{1}{2} \leqq R\left(\omega_{\Downarrow}\right) \leqq \frac{1}{2}(n-1)
$$

and more exactly

$$
\omega_{1} \leqq \frac{1}{2}(n-1) \quad \text { and } \quad\left|\omega_{\nu}\right| \leqq\left(\frac{n(n-1)}{2 \nu}\right)^{1 / 2} \text { for } \nu \geqq 2 .
$$

Proof. Let $B$ be the symmetric matrix $\frac{1}{2}\left(A+A^{\prime}\right)$. All its main diagonal elements are zeros and all other elements equal $\frac{1}{2}$. Since $B$ is a generalized stochastic matrix with row-sum $\frac{1}{2}(n-1)$, its greatest root is $\frac{1}{2}(n-1)$. Moreover, it follows from [3] that the nontrivial roots remain unchanged if we subtract from all the elements of each column the number $\frac{1}{2}$. We obtain the diagonal matrix $D\left(-\frac{1}{2},-\frac{1}{2}, \cdots,-\frac{1}{2}\right)$. Hence $B$ has the root $\frac{1}{2}(n-1)$ and $n-1$ roots $-\frac{1}{2}$.

In 1902, I. Bendixson [1] proved the following theorem.

Let $T$ be a matrix with real elements, $S$ the symmetric matrix $\frac{1}{2}\left(T+T^{\prime}\right)$, and $M$ and $m$ the maximum and the minimum of the char-

${ }^{1}$ Research sponsored by the Air Force Office of Scientific Research, Office of Aerospace Research, United States Air Force, under AFOSR Grant number 982-67. 
acteristic roots of $S$, respectively, then the real part of any root $\eta_{\nu}$ of $T$ satisfies

$$
m \leqq R\left(\eta_{v}\right) \leqq M
$$

Applying this theorem to the matrix $A$ it follows that

$$
-\frac{1}{2} \leqq R\left(\omega_{\nu}\right) \leqq \frac{1}{2}(n-1) .
$$

Since $A$ is nonnegative, it follows from the theorem of Frobenius (see [4]) that $\omega_{1}$ is positive, hence by (1)

$$
0<\omega_{1} \leqq \frac{1}{2}(n-1),\left|\omega_{\nu}\right| \leqq \frac{1}{2}(n-1) \quad \text { for } \nu \geqq 2,
$$

and it follows from (1) that

$$
-\frac{1}{2} \leqq R\left(\omega_{\nu}\right) \leqq\left|\omega_{\nu}\right| \leqq \frac{1}{2}(n-1) .
$$

It can easily be seen that this result cannot be improved in general.

Let $n$ be an odd integer and let us assume that each of the $n$ players wins $\frac{1}{2}(n-1)$ games. Then the tournament matrix is a generalized stochastic matrix with row-sum $\frac{1}{2}(n-1)$, hence $\omega_{1}=\frac{1}{2}(n-1)$. Since the trace of $A$ is zero, the sum of the real parts of all the roots different from $\omega_{1}$ must be $-\frac{1}{2}(n-1)$, and by $(2) R\left(\omega_{\nu}\right)=-\frac{1}{2}$ for $\nu=2,3, \cdots, n$.

For $\nu \geqq 2$ the inequality (2) can be improved.

It follows from a theorem of I. Schur [6] that

$$
\left|\omega_{1}\right|^{2}+\left|\omega_{2}\right|^{2}+\cdots+\left|\omega_{n}\right|^{2} \leqq \frac{1}{2} n(n-1) \text {. }
$$

Hence

$$
\begin{gathered}
\nu\left|\omega_{\nu}\right|^{2} \leqq\left|\omega_{1}\right|^{2}+\left|\omega_{2}\right|^{2}+\cdots+\left|\omega_{\nu}\right|^{2} \leqq \frac{1}{2} n(n-1), \\
\left|\omega_{\nu}\right| \leqq\left(\frac{n(n-1)}{2 \nu}\right)^{1 / 2} .
\end{gathered}
$$

It follows in particular that if $A$ has imaginary roots, then

$$
\left|I\left(\omega_{\nu}\right)\right| \leqq\left(\frac{n(n-1)}{6}\right)^{1 / 2}
$$

\section{REFERENCES}

1. Ivar Bendixson, Sur les racines d'une equation fundamentale, Acta Math. 25 (1902), 359-366.

2. Alfred Brauer, Limits for the characteristic roots of a matrix. II, Duke Math. J. 14 (1941), 21-26.

3. - $A$ new proof of theorems of Perron and Frobenius on nonnegative matrices, Duke Math. J. 24 (1957), 367-368; A method for the computation of the greatest root of a nonnegative matrix, SIAM J. Numer. Anal. 3(1966), 564-569. 
4. - Limits for the characteristic roots of a matrix. IV, Duke Math. J. 19 (1952), 75-91.

5. H. J. Ryser, "Matrices of zeros and ones in combinatorial mathematics," in Recent advances in matrix theory edited by Hans Schneider, University of Wisconsin Press, Madison, Wisconsin 1964.

6. I. Schur, Über die charakteristischen Wurzeln einer linearen Substitution mit einer Anwendung auf die Theorie der Integralgleichungen, Math. Ann. 66 (1909), 488510.

\title{
WAKE Forest UNIVERSITY
}

\section{A GENERAL MEAN VALUE THEOREM}

\author{
BY E. D. CASHWELL AND C. J. EVERETT
}

Communicated by Jürgen K. Moser, May 17, 1968

We present here in general terms the idea of the mean of a function relative to a "weight function" $w(\xi, \nu)$, special instances and applications appearing elsewhere [1], [2].

1. The weight function. If $X=[h, k]$ is a real interval, $(I, A, \mu)$ a finite measure space with $\mu(I)=1$, and $w(\xi, \nu)$ a nonnegative function on $X \times I$ which, for each $\nu$ of $I$, is measurable, and positive a.e. on $X$, then the indefinite integral

$$
W(x, \nu)=\int_{h}^{x} w(\xi, \nu) d \xi
$$

is defined on $X \times I$, and the function

$$
W(x)=\int_{I} W(x, \nu) d \mu, \quad x \in X
$$

which we assume to exist, is continuous and strictly increasing on $X$, as is $W(x, \nu)$ for each $\nu$.

2. The mean of a function. Let $x(\nu)$ be any $\mu$-integrable function on $I$ to $X$ for which the integral functional

$$
W_{x}=\int_{I} W(x(\nu), \nu) d \mu
$$

exists. Let $x_{u}$ be the essential upper bound of $x(\nu)$ on $I$, i.e., the g.l.b. of all real $x$ for which $\mu\{\nu \mid x(\nu)>x\}=0$, the essential lower bound

${ }^{1}$ Work performed under the auspices of the U. S. Atomic Energy Commission. 